Revista Universo Contábil, ISSN 1809-3337

Blumenau, v. 7, n. 3, p. 06-24, jul./set., 2011

doi:10.4270/ruc.2011319

Disponível em www.furb.br/universocontabil

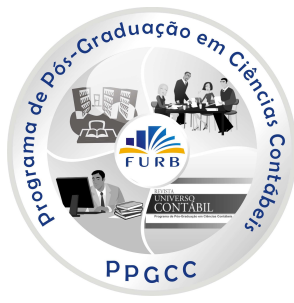

ADOÇÃO DE SISTEMAS DE CUSTOS NO SETOR PÚBLICO: REFLEXÕES SOBRE A LITERATURA NACIONAL VEICULADA EM PERIÓDICOS ACADÊMICOS 1

\title{
THE ADOPTION OF COSTS SYSTEMS IN THE PUBLIC SECTOR: REFLEXIONS ON THE BRAZILIAN LITERATURE PUBLISHED IN ACADEMIC JOURNALS
}

\author{
Eugenio José Silva Bitti \\ Doutorando em Engenharia de Produção pela \\ Escola Politécnica da Universidade de São Paulo \\ Endereço: Av.Prof. Almeida Prado, Travessa 2, n.128, Ed. Biênio, 2o. andar \\ CEP: 05508-900 - São Paulo/SP - Brasil \\ E-mail: ebitti@usp.br \\ Telefone: +55 11 8609-6243 \\ André Carlos Busanelli de Aquino \\ Doutor em Ciências Contábeis pela FEA/USP \\ Professor da Faculdade de Economia, Administração e Contabilidade \\ de Ribeirão Preto da Universidade de São Paulo \\ Endereço: Av. Bandeirantes, 3900, Monte Alegre \\ CEP: 14040-905 - Ribeirão Preto/SP - Brasil \\ E-mail: aaquino@usp.br \\ Telefone: +55 16 3602-4972 \\ Ricardo Lopes Cardoso \\ Doutor em Ciências Contábeis pela FEA/USP \\ Professor da Escola de Administração Pública e de Empresas da FGV/RJ \\ Professor da Faculdade de Administração e Finanças da UERJ \\ Endereço: Praia de Botafogo, 190, sala 526, Botafogo \\ CEP: 22250-090 - Rio de Janeiro/RJ - Brasil \\ E-mail: ricardo.lopes.cardoso@fgv.br \\ Telefone: +55 $213799-5711$
}

\section{RESUMO}

O presente artigo realiza uma análise temática dos estudos sobre a aplicação de sistemas de custos no setor público brasileiro. Passados mais de 10 anos da publicação dos clássicos Gupta, Dirsmith e Fogarty (1994), Geiger e Ittner (1996) e Ittner e Larker (1998), a adoção e

\footnotetext{
1 Artigo recebido em 04.07.2010. Revisado por pares em 14.08.2010. Reformulado em 19.09.2010. Recomendado para publicação em 22.09.2010 por Ilse Maria Beuren (Editora). Publicado em 30.09.2011. Organização responsável pelo periódico: FURB.
} 
o uso de sistemas de custos no setor público têm recebido crescente atenção na literatura internacional, que se propõe, prioritariamente, a explicar a adoção dos sistemas e seus impactos nas organizações. Em geral, os artigos publicados no exterior utilizam levantamentos (surveys) baseados em abordagens teóricas pluralistas (contingenciais e institucionais). Os resultados da pesquisa nacional são aqui representados pelos artigos publicados em periódicos listados na base Qualis/CAPES, sendo que somente sete artigos foram identificados. Os resultados da análise temática indicam que os estudos publicados no Brasil, ao contrário da tendência estrangeira, utilizam estudos de caso descritivos ou propõem modelos prescritivos de gestão e mensuração de custos; adicionalmente, desconsideram os achados de estudos empíricos anteriores. Conclui-se que, por estas características, torna-se difícil consolidar e comparar resultados, assim como construir teorias no contexto brasileiro. Espera-se que essa constatação permita a reflexão e o amadurecimento da pesquisa nacional sobre a temática da aplicação de sistemas de custos no setor público brasileiro.

Palavras-chave: Custos. Contabilidade pública. Setor público. Periódicos acadêmicos.

\section{ABSTRACT}

This paper consists on a thematic analysis about the implementation of costs accounting systems in the Brazilian public sector. Ten years after the publication of classics as Gupta, Dirsmith and Fogarty (1994), Geiger and Ittner (1996), and Ittner and Larker (1998), the adoption and use of costs systems in the public sector is receiving an increasing attention in the international academic arena, which main purpose is to explain the adoption of costs systems and their impacts on organizations. Generally, the papers published abroad use surveys based on a plural theoretical approach (contingency and institutional theories). We considered papers published on Brazilian academic journals listed in the Qualis/CAPES as a proxy of the Brazilian research on this field, only seven papers were identified. Results from the thematic analysis show that the papers published in Brazilian, different from the international trend, are based on descriptive case studies or prescribe management and costs measured methods; in addition, they do not consider the findings of previous empirical researches. All these features make it difficult to consolidate and compare the results and to build theories in the Brazilian context. We expect that these findings contribute the reflexion about and improvement of the Brazilian research in this field.

Keywords: Costs. Governmental accounting. Public sector. Academic journals.

\section{INTRODUÇÃO}

O projeto e a adoção de sistemas de custos é um dos temas mais discutidos e pesquisados em contabilidade gerencial. Sistemas de custos oferecem uma medida dos recursos consumidos no cumprimento de um propósito específico, seja a execução de um serviço, a geração de um produto ou mesmo condução de um projeto ou programa, independentemente da fonte de financiamento.

Sistemas de controle gerencial de custos potencialmente atendem a duas demandas organizacionais básicas: informação para tomada de decisão e controle do comportamento de agentes (MERCHANT; SIMON, 1986; ZIMMERMAN, 1997; MARTINS, 2006). Enquanto que para decisão, a informação de desempenho reduz a improvisação dos agentes na escolha entre conjuntos alternativos de ação. Em termos de controle, o detalhamento, a acurácia e a tempestividade da informação tendem a mitigar o risco moral (PIZZINI, 2006).

Apesar da consistente literatura sobre a aplicação de artefatos gerenciais no setor 
privado em diversos contextos organizacionais, somente mais recentemente a melhoraria de desempenho de organizações públicas tem recebido atenção por parte da pesquisa em controles gerenciais (CAVALLUZZO; ITTNER, 2004).

O setor público representa um conveniente laboratório para testes de predições teóricas em torno do surgimento, desenvolvimento e aplicação de sistemas gerenciais de custos. A estrutura de custos das organizações de Estado tende a ser complexa, incluindo um vasto rol tanto de direcionadores de custos quanto de objetos de custeio, muitas vezes sem que a figura do preço final do produto gerado seja caracterizada (BJORNENAK, 2000). Com isso, o próprio papel estratégico do órgão permanece obscuro, o que demanda um nível de análise que muitas vezes é inacessível para a gerência operacional destes órgãos.

Se comparado aos avanços vistos nos Estados Unidos, Austrália, Nova Zelândia e dos Estados que constituem a União Européia (sobretudo no Reino Unido), entende-se que no Brasil ainda se está no início do processo de entendimento da formação de modelos gerenciais de custos no setor público. Trabalhos anteriores justificam a tênue literatura nacional veiculada em periódicos científicos com o fato de que apenas recentemente a cultura de custos passou a estar mais presente nos organismos de governo (ALONSO, 1999; SILVA, 2007) e este quadro não apresenta sinais de alteração (ALMEIDA; BORBA; FLORES, 2009).

Não obstante, observa-se iniciativas de implantação de sistemas de custos nas unidades de governo. No nível federal: as forças armadas, institutos de pesquisa (como a Embrapa), ministérios (como o da Educação) e hospitais (como o Inca), já possuem seus sistemas de informação de custos em nível avançado em relação à realidade nacional. Já no nível municipal, Prefeituras Municipais de Guarulhos - SP, Salvador - BA, Rio de Janeiro RJ, apenas para citar alguns exemplos, já possuem aplicações para alguns de seus serviços e áreas. A observação destas iniciativas pode ser sinal de percepção de utilidade da informação de custos pelos usuários destes órgãos.

Entretanto, em alguns desses entes a experiência de apuração de custos se iniciou (ou se limita a) com objetos de custeio relacionados a divisões (programas, atividades, serviços etc.) que prestam serviços a clientes externos e que estavam com algum contingenciamento ou pressão orçamentária, ou ainda submetidos à imposição para implantação de controles. Podese citar a Prefeitura do Rio, que começou a implantar o sistema de custos pela Comlurb (empresa de limpeza urbana) no período que o sindicato dos motoristas estava promovendo greves, então, a entidade precisou decidir entre manter a frota verticalizada ou terceirizá-la, para isso demandou informação de custos.

Outro exemplo é a Marinha, cujos sistemas de apuração de custos estão mais desenvolvidos nas unidades classificadas como Organizações Militares Prestadoras de Serviços (OMPS). A Lei $\mathrm{n}^{\circ}$ 9.724/1998 (art. 1º, inciso V) estabelece, entre outros atributos, que unidades da Marinha apurem seus "custos por processo contábil específico". A fim de que possam ser classificadas como OMPS tal classificação era apresentada pela referida lei como condição sine qua non para se habilitarem a firmar contrato de gestão com a administração superior.

A simples demanda legal - existente desde 1964 (Lei no 4.320) - não se caracteriza como incentivo suficiente para implantação e efetivo uso de tais sistemas de forma abrangente no país. Foram necessárias iniciativas como: (i) a do Tribunal de Contas da União (TCU) que emitiu o Acórdão 1.078/2004 recomendando, formalmente, que a Lei de Responsabilidade Fiscal (LRF, Lei Complementar $n^{\circ}$ 101/2000) fosse respeitada no tocante à apuração de custos na administração pública federal; (ii) a Portaria Interministerial $n^{\circ} 945 / 2005$ que criou o Comitê Interministerial de Custos; e (iii) a rodada de intensas discussões promovidas em 2009 pelo Ministério da Fazenda, na sede da Escola de Administração Fazendária (ESAF).

Essas discussões culminaram com a definição, pelo Ministério da Fazenda, dos parâmetros para captação dos dados relativos à liquidação de despesas disponíveis nos 
sistemas estruturantes e dos procedimentos para ajustá-los ao regime de competência e transformá-los em informações de custos. Neste movimento, a adoção e uso de sistemas de custos no Brasil - antes apenas um fenômeno carente de estudos - ganha relevância social e, sobretudo, a dinâmica e razões da adoção e uso passa a ser relevante enquanto objeto de investigação científica.

Assim, dada a emergente literatura internacional em sistemas de custos no setor público, empírica em sua grande maioria, e a crescente relevância prática da adoção destes sistemas no Brasil, questionam-se: Como tem sido conduzida a pesquisa nacional em torno da adoção de sistemas de custo no setor público? Qual o perfil metodológico destes estudos? Qual fundamentação teórico-conceitual tem orientado os pesquisadores?

A produção científica da área de contabilidade no Brasil ainda está por se consolidar, se comparada à internacional. Em termos de escolhas metodológicas, Theóphilo e Iudícibus (2005) estudaram a produção científica da área de contabilidade no Brasil no período de 1994 a 2003. A análise mostra um incremento de estudos empíricos (em substituição aos estudos teóricos ou puramente didáticos), e de estudos positivos (em substituição aos estudos normativos, prescritivos). Contudo este predomínio ainda é localizado em alguns periódicos e centros de pesquisa (CARDOSO; OYADOMARI; MENDONÇA NETO, 2007; SILVA; OLIVEIRA; RIBEIRO FILHO, 2005; OLIVEIRA, 2002).

A área ainda é caracterizada por trabalhos sem passado, pois mais da metade dos trabalhos no período de 1999 a 2003 não fazia referências a estudos anteriores sobre o mesmo tema/assunto abordado (THEÓPHILO; IUDÍCIBUS, 2005). Por fim, a excessiva quantidade de estudos exploratórios indica a falta de continuidade dos estudos anteriores. Essa baixa capacidade de sistematização de resultados anteriores dificulta a construção do campo de conhecimento (ZIMMERMAN, 2001; LUKKA; MOURITSEN, 2002; HOPWOOD, 2002; LUFT; SHIELDS, 2002).

Com a motivação de elucidar a baixa sistematização dos resultados das pesquisas nacionais no tema, o presente artigo procurou retratar as contribuições da pesquisa nacional, as regularidades em abordagens teóricas e em estratégias de pesquisa adotadas pelos autores. Com o uso de técnicas de demarcação de referencial teórico, partindo de periódicos nacionais listados na Qualis/CAPES, foram levantados os trabalhos que de alguma forma divulgavam resultados de esforços de pesquisa no tema. Através de uma Análise Temática em cada trabalho, foram identificadas as dimensões teórica, metodológica e técnica presentes. As regularidades e tendências observadas foram comparadas com aquelas encontradas na literatura internacional no tema. Para dar evidências da baixa sistematização de resultados, investigaram-se as características das referências bibliográficas dos trabalhos nacionais, contudo tal iniciativa não é suficiente para caracterizar este estudo como bibliométrico.

O pouco volume de trabalhos teórico-empíricos na literatura nacional no tema é notável. Assim como a não consideração de estudos anteriores ou teorias. Por sua vez, na literatura estrangeira verifica-se o uso de teorias, como Teoria da Contingência e a Teoria Institucional, aplicadas cada vez mais de forma conjunta (complementaridade teórica na construção de hipóteses). No caso brasileiro, os estudos empíricos identificados indicam ausência de orientação teórica, resignando-se a estudos de caso único em organismos (principalmente hospitais públicos) que passaram por projetos de implantação de ferramentas de custeio (principalmente o Custeio Baseado em Atividades - ABC). Embora estudos deste tipo sejam úteis para disseminar o ambiente organizacional, o desenho de tais artefatos e a prática no uso da contabilidade de custos, eles não são capazes de gerar evidências de alcance mais amplo quanto às motivações e objetivos de sua aplicação (GEIGER; ITTNER, 1996).

Na continuação, este artigo está organizado como segue: na seção 2 são apresentados os antecedentes teóricos extraídos da literatura internacional sobre a adoção de sistemas de informações gerenciais no setor público. Na seção 3, é apresentada a estratégia de coleta de 
dados e o critério de análise dos artigos nacionais no tema. Na seção seguinte é feita a análise destes artigos nacionais. Por fim, são feitas as considerações finais e propostos alguns direcionamentos para pesquisas futuras.

\section{BREVE APRESENTAÇÃO DAS PESQUISAS INTERNACIONAIS}

O propósito desta seção não é fazer uma análise crítica da produção acadêmica internacional (para tanto, sugere-se BHIMANI, 1996; SHIELDS, 1998; YAKHOU; DORWEILER, 1995). Mas apresentar algumas características (positivas) da produção acadêmica internacional sobre a implantação de sistemas de informação gerencial para, a partir disso, analisar criticamente a literatura nacional.

O setor público tem sido alvo de discursos em prol da adoção de tecnologias de contabilidade gerencial desenvolvidas no setor privado, principalmente por pressões exercidas pelo governo central (LAPSLEY; WRIGHT, 2004). A discussão teórica mais recorrente na literatura internacional procura explicar se a adoção de sistemas de custos decorre de demanda organizacional ou de efeitos institucionais. Enquanto a demanda organizacional é gerada pela percepção da utilidade e relevância da informação de custos por parte de gestores, os efeitos institucionais podem vir da busca por legitimação das práticas gerenciais perante grupos de interesse externos à organização. A literatura subjacente mostra que estas duas hipóteses se complementam (GUPTA; DIRSMITH; FOGARTY, 1994; GEIGER; ITTNER, 1996).

Os sistemas de informação de custos são mais voltados à decisão ou ao controle, dependendo das características ambientais às quais a organização está sujeita. A literatura de teoria da contingência (CHENHALL, 2003) prevê certa aderência entre o desenho dos sistemas de custos e: (i) a estratégia escolhida pela organização (LANGFIELD-SMITH, 1997), (ii) os determinantes estruturais (como mix de serviços, porte, entidade controladora) (LAWRENCE, 1990) e (iii) o ambiente competitivo (HILL, 2001). Complementarmente, as exigências impostas por tarefas técnicas incentivam a adoção de meios para coordenar atividades internas, o que demanda geração e fluxo de informação acurada (GUPTA; DIRSMITH; FOGARTY, 1994).

Esse contingenciamento não está ligado apenas a aspectos técnicos internos, mas também à incerteza ambiental externa. Isto é, se a organização atua em ambientes caracterizados por forte competição, espera-se que o perfil dos controles gerenciais aplicados seja relativamente mais rígido. O paradigma atual da gestão pública estabelece um quadro de necessária auto-sustentabilidade e competição por recursos (caracterizados pela dotação orçamentária) por parte de órgãos públicos. Esse paradigma sujeita a entidade pública a pressões semelhantes às observadas no setor privado (BROWN; SPROGHE, 1987). Por exemplo, ao analisar a forma como o gerenciamento de custos ocorre em agências federais norte-americanas, Geiger e Ittner (1996) observam que quanto maior a incerteza em relação ao fluxo de recursos para sua manutenção (incluindo o orçamento repassado pela agência central) mais intensa é a utilização de sistemas de custos por parte destes órgãos.

Embora se reconheça a importância de aspectos técnicos relativos às atividades executadas e das questões ambientais em torno da organização, considera-se que tais impactos podem ser secundários quando comparados ao nível de comprometimento da alta gerência no tocante à implantação de sistemas gerenciais. Além do comprometimento, a autoridade da tomada de decisão e o treinamento em técnicas de avaliação de desempenho têm influência significantemente positiva sobre o desenvolvimento e uso destes indicadores (CAVALLUZZO; ITTNER, 2004). Contudo, mesmo na esfera privada, a implantação de inovações gerenciais encontra resistência ao contrariarem o sistema de crenças vigente na organização (ITTNER; LARCKER 1998).

Por fim, eventuais problemas em sistemas de informação e dificuldades em selecionar e interpretar métricas de desempenho apropriadas em situações de alto subjetivismo exercem 
importante papel na implementação e uso do sistema (LAPSLEY; WRIGHT, 2004). Apesar de o tema subjetividade da avaliação ser bem estudado no âmbito da iniciativa privada (HARTMANN, 2000), as evidências dos efeitos no setor público ainda são escassas, como os benefícios percebidos das recentes regras de avaliação de desempenho implantadas pelo governo americano.

$\mathrm{Na}$ perspectiva da Teoria Institucional, as organizações obtêm legitimação ao atenderem expectativas externas. Uma secretaria, agência reguladora ou qualquer ente público obtém legitimidade por se conformar às expectativas externas de práticas aceitáveis, mesmo que no âmbito interno tais técnicas sejam modificadas ou ignoradas para fins gerenciais. Uma organização, assim, poderá formalmente adotar elementos destes modelos organizacionais arquetípicos para demonstrar racionalidade quanto ao controle de suas operações, mesmo que tais práticas não contribuam à eficiência alocativa de seus recursos (GUPTA; DIRSMITH; FOGARTY, 1994). Organizações que dependem de legitimação para manter sua dotação orçamentária apresentariam baixa resistência em adotar os sistemas exigidos pelo órgão de controle, ainda que não fosse considerada para fins gerenciais (GEIGER; ITTNER, 1996).

Geiger e Ittner (1996) confirmam que métricas de custeio implantadas por determinação estatutária em secretarias da administração federal nos EUA não são efetivamente usadas para fins gerenciais internos. Da mesma forma, Ittner e Larcker (1998) concluíram que o caráter compulsório da iniciativa norte-americana para aumentar a governança em agências federais (Government Performance and Results Act, 1993) apesar de promover sua adoção em diversas agências e níveis, o uso das informações geradas em muitos casos foi destinado à mera prestação de contas ao órgão de controle do orçamento federal.

A literatura de sistemas gerenciais tem identificado fortes complementaridades entre as correntes teóricas acima mencionadas. É o que indica o fato de certas práticas surgirem específicas e contextualizadas e, dados os resultados, tornarem-se institucionalizadas ao longo do tempo (DRAZIN; VAN de VEN, 1985). Tais técnicas nasceriam idiossincráticas à organização, atendendo expectativas externas e, com o tempo, certo isomorfismo mimético as difundiria, mesmo em detrimento das necessidades internas à organização (DIMAGGIO; POWELL 1983).

A complementaridade das duas perspectivas também pode ser verificada quando os mecanismos de controle empregados são desmembrados em dois conjuntos relativamente distintos: o primeiro, simbólico, para atender expectativas externas; e o segundo efetivamente empregado para atender às reais necessidades gerenciais. Um exemplo é o emprego, por entidades privadas brasileiras, do custeio por absorção para atender exigências do fisco e da legislação societária. É consenso que a alocação de overheads por critérios subjetivos ou arbitrários tem efeitos danosos sobre a informação gerencial e, conseqüentemente, para a gestão. Assim, por força de lei, as empresas apuram custos pelo método do custeio por absorção para mensurar seus estoques e determinar o CMV (custo das mercadorias vendidas) e o lucro objeto de tributação, ao mesmo tempo em que, parte delas, aplica outras metodologias menos subjetivas ( custeio direto, variável e/ou $\mathrm{ABC}$ ) para fins gerenciais.

Mais recentemente, abordagens advindas das novas teorias da firma têm buscado entender a dinâmica de formação destes dispositivos gerenciais. A discussão em torno de mecanismos de controle ambientados na Economia dos Custos de Transação sugere que estruturas de controle gerencial podem ser analisadas como contratos (implícitos ou explícitos, formais ou informais) entre a organização e seus membros, governando as contribuições destes agentes (SPEKLÉ, 2001). Nesta abordagem, a opção por uma configuração específica de estrutura de controle seria função do nível de aderência desta estrutura com o tipo de atividades desenvolvidas pela organização. 


\section{DESENHO DA PESQUISA}

O desenvolvimento de determinada área de conhecimento através de análises temáticas em revisões sistemáticas de literatura possibilita identificar não só as tendências da área, mas também permite direcionar os esforços para preencher lacunas teóricas ou mesmo corrigir vieses metodológicos de determinada área.

$\mathrm{Na}$ literatura internacional observa-se o crescimento de tais estudos associados ao tema de contabilidade, envolvendo mapeamento de temas e abordagens teóricas, metodológicas (ITTNER; LARCKER, 2001, 2002; LUFT; SHIELDS, 2003), avaliação e ranking de periódicos acadêmicos (BROWN; GARDNER; VASARHELY, 1987; HULL; WRIGHT, 1990), críticas sobre os rumos da academia (BAMBER; CRISTENSEN; GAVER, 2000; LUKKA; GRANLUND, 2002; REITER; WILLIAMS, 2002), críticas sobre lacunas e vieses na pesquisa em determinada área (ZIMMERMAN, 2001; HOPWOOD, 2002; LUFT; SHIELDS, 2002; LUKKA; MOURITSEN, 2002) e construção de redes sociais e divulgação de conhecimento (BROWN, 2005).

No Brasil, na área de contabilidade, os estudos epistemológicos predominam sob a forma de estudos bibliométricos, buscando regularidades em abordagens metodológicas (THEÓPHILO; IUDÍCIBUS, 2005, CARDOSO et al., 2005), temas (RICCIO; CARASTAN; SAKATA, 1999; FREZATTI, 2000; OLIVEIRA, 2002; CARDOSO et al., 2005; SILVA; OLIVEIRA; RIBEIRO FILHO, 2005) e filiação acadêmica de autores (CARDOSO et al., 2005; SILVA; OLIVEIRA; RIBEIRO FILHO, 2005). Os estudos bibliométricos na área, no Brasil, incluem na amostra trabalhos veiculados em congressos e discutem com isso tendências em uma área (CARDOSO; OYADOMARI; MENDONÇA NETO, 2007), outros consideram apenas periódicos buscando a existência de redes sociais de cooperação científica ou a predominância de escolhas metodológicas em certo tema (OLIVEIRA, 2002; SILVA; OLIVEIRA; RIBEIRO FILHO, 2005).

Análises temáticas são pouco freqüentes, um exemplo pode ser visto em Theóphilo e Iudícibus (2005). Como o objetivo do presente estudo é evidenciar as contribuições da pesquisa nacional ao tema, as teorias desafiadas e os métodos empregados, assim como discutir sistematização dos resultados, optou-se pelo uso de análise temática nos estudos publicados em periódicos acadêmicos. A análise temática não considerou trabalhos publicados em anais de congressos, dissertações de mestrado, teses de doutorado e livredocência, nem livros didáticos. A razão para a não consideração destes trabalhos é que mais representam tendências de pesquisa na área, do que projetos finalizados e consolidados.

A contagem (bibliométrica) de estudos publicados em congressos aponta tendências, motivações de pesquisadores em eventos científicos; enquanto contagens em dissertações e teses, a dinâmica nos programas de pós-graduação e formação de grupos de pesquisa. Dado que se procura as contribuições efetivas em termos de achados (sejam recomendações de aplicações ou testes de teorias), observaram-se resultados que foram submetidos pelos seus próprios autores ao processo competitivo de publicação, obtendo certa legitimação da pesquisa conferida pelos referees destes veículos.

Sendo o objetivo central deste trabalho a sumarização das contribuições dos estudos da temática no caso brasileiro, e busca por regularidades em abordagens teóricas e de estratégias de pesquisa que suportam tais estudos, restringiu-se o universo dos trabalhos àqueles publicados em fontes nas quais o processo competitivo de avaliação referendasse os resultados divulgados. Para demarcação da pesquisa nacional existente, adotou-se o método de construção de referencial teórico descrito em Aquino, Pagliarussi e Bitti (2008).

Inicialmente, foi utilizado o acervo de periódicos do sistema de referência Scielo (Scientific Electronic Library Online), seguindo Murcia e Borba (2008). Utilizou-se uma única palavra-chave ("custos"), restringindo-se a busca aos periódicos da grande área "Ciências Sociais Aplicadas". Esta base reúne periódicos nacionais em formato eletrônico, 
assim como produz e publica indicadores do seu uso e impacto (PACKER et al., 1998). Um total de 121 artigos foi inicialmente selecionado. Pela leitura dos resumos (abstracts), os artigos foram classificados quanto à relação direta com o tema "aplicação de sistemas gerenciais de custos no setor público". Dos 121 artigos, apenas três artigos estavam diretamente relacionados à aplicação de ferramental de custos no setor público.

Contudo, a maior parte das pesquisas nacionais é divulgada em periódicos nãoindexados nesta base. Portanto, a exemplo de outros estudos na área (THEÓPHILO; IUDÍCIBUS, 2005; CARDOSO; OYADOMARI; MENDONÇA NETO, 2007) a busca foi estendida para os periódicos listados no Qualis/CAPES (Coordenação de Aperfeiçoamento de Pessoal de Nível Superior), abrangendo a área de Ciências Sociais Aplicadas. Em 72 periódicos, incluindo a Revista do Serviço Público, outros quatro trabalhos foram identificados. A busca na literatura nacional publicada em periódicos resultou, portanto, em sete trabalhos publicados em periódicos científicos até 31 de agosto de 2010.

\section{A LITERATURA NACIONAL}

Esta seção analisa a literatura nacional dedicada à demanda por, implantação ou uso de sistemas de informações gerenciais no setor público brasileiro. Inicialmente são apresentados comentários sobre as características gerais desse conjunto de produções bibliográficas; na sequência são apresentadas críticas aos artigos em comparação com a produção internacional.

\subsection{Comentários preliminares}

No cenário brasileiro chama atenção a atração exercida pelo setor de saúde (quatro trabalhos). Cinco trabalhos são empírico-descritivos, sendo três estudos de caso e dois levantamentos. $\mathrm{O}$ fato de que a maioria dos estudos identificados foi publicada nos últimos dois anos sugere duas interpretações: (i) pouca atenção vinha sendo dada ao tema, seja por pesquisadores, editores ou revisores de periódicos e/ou (ii) uma possível baixa relevância percebida em relação ao fenômeno sistemas de custos pelos agentes do setor público no Brasil. Neste caso os efeitos dos impactos da LRF, dentre eles o citado acórdão do TCU, podem concorrer para alavancar o interesse sobre o tema.

Não desejando fazer apologia à escola positiva de pesquisa em contabilidade, em detrimento da normativa, destaca-se um ponto comum aos artigos analisados: a potencial dificuldade de generalização dos achados. Esta discussão é similar ao debate ocorrido entre os anos de 2001 e 2003, iniciado por Zimmerman (2001), que afirmou a época que a pesquisa em Contabilidade Gerencial não conseguia produzir um corpo coerente de conhecimentos, ou seja, tinha dificuldade em acumular sistematicamente achados e conclusões sobre o fenômeno. Diversas críticas foram feitas a várias afirmações em Zimmerman (2001), entre elas Lukka e Mouritsen (2002), Hopwood (2002) e Luft e Shields (2002).

Entretanto, nenhuma delas negou a importância da não sistematização dos achados para o desenvolvimento do campo de conhecimento. Entre os motivos que Zimmerman cita para esta dificuldade em construir conhecimento, estão o constante uso da justificativa de ausência de dados para a não realização de estudos empíricos, o que seria um indício da pouca criatividade dos pesquisadores no tema. $\mathrm{O}$ excessivo uso de abordagem indutiva, que não baseada em teoria, procura apenas descrever e documentar as práticas gerenciais adotadas.

Por fim, o uso de abordagens teóricas não falseáveis e, portanto, não passíveis de poder preditivo, impediria a comparabilidade dos resultados. No artigo em que criticam parte das considerações de Zimmerman (2001), Lukka e Mouritsen (2002) defendem que a generalização dos resultados em Contabilidade Gerencial se daria através da comparação de estudos empíricos, sejam descritivos ou explicativos, relacionando-os. Desta forma emergiriam novas proposições falseáveis. A mesma discussão aplicada à literatura de 
Contabilidade Gerencial, direcionado às organizações do setor privado, também se aplica à adoção de sistemas no setor público.

Não se encontra essa realidade nos estudos analisados. A maior parte das citações e referências é derivada de (i) normas contábeis ou definições contábeis extraídas de livros textos, (ii) prescrições acerca de sistemas de custos, e (iii) estudos ainda não publicados em periódicos nacionais ou internacionais (Quadro 1).

\begin{tabular}{|c|c|c|c|c|c|}
\hline $\begin{array}{l}\text { Autor } \\
\text { (ano) }\end{array}$ & $\begin{array}{c}\text { Abordagem } \\
\text { metodológica* }\end{array}$ & Principais achados & $\begin{array}{l}\text { Unidade de } \\
\text { análise }\end{array}$ & $\begin{array}{c}K \\
(\%) \\
* *\end{array}$ & $\begin{array}{l}L \\
(\%) \\
* * *\end{array}$ \\
\hline $\begin{array}{l}\text { Alonso } \\
(1999)\end{array}$ & Prescritivo & $\begin{array}{l}\text { Recomenda a aplicação do Activity } \\
\text { Based Costing nas unidades do governo } \\
\text { federal visando uma maior capacidade } \\
\text { gerencial do governo. }\end{array}$ & $\begin{array}{l}\text { Administração } \\
\text { Federal } \\
\text { (Não específico) }\end{array}$ & 5 & 0 \\
\hline $\begin{array}{l}\text { Miranda } \\
\text { et al. } \\
(2007)\end{array}$ & $\begin{array}{l}\text { Empírico } \\
\text { Descritivo }\end{array}$ & $\begin{array}{l}\text { O Activity Based Costing é difundido, } \\
\text { mas pouco aplicado devido à } \\
\text { complexidade do modelo e pela baixa } \\
\text { relação custo-benefício de um projeto de } \\
\text { implantação do ABC. }\end{array}$ & $\begin{array}{l}\text { Setor Hospitalar } \\
\text { (Hospitais } \\
\text { universitários) }\end{array}$ & 18 & 0 \\
\hline $\begin{array}{l}\text { Lagioia et } \\
\text { al. (2008) }\end{array}$ & $\begin{array}{l}\text { Empírico } \\
\text { Descritivo }\end{array}$ & $\begin{array}{l}\text { A padronização de atividades e } \\
\text { processos gerou ganhos em desempenho } \\
\text { na organização analisada. }\end{array}$ & $\begin{array}{l}\text { Setor Hospitalar } \\
\text { (Estudo de caso } \\
\text { único) }\end{array}$ & 56 & 89 \\
\hline $\begin{array}{l}\text { Silva, } \\
\text { Davis e } \\
\text { Viveiros } \\
(2008) \\
\end{array}$ & $\begin{array}{l}\text { Empírico } \\
\text { Descritivo }\end{array}$ & $\begin{array}{l}\text { Aumento da qualidade e relevância da } \\
\text { informação de custos decorrente do } \\
\text { redesenho das unidades administrativas } \\
\text { da Marinha. }\end{array}$ & $\begin{array}{l}\text { Administração da } \\
\text { Marinha do Brasil }\end{array}$ & 13 & 0 \\
\hline $\begin{array}{l}\text { Luque et. } \\
\text { al. (2008) }\end{array}$ & Presc & $\begin{array}{l}\text { Recomenda a aplicação de um sistema } \\
\text { de custeio baseado nas contas de custo } \\
\text { corrente do governo: pessoal da ativa e } \\
\text { insumos intermediários. Os principais } \\
\text { benefícios seriam a maior capacidade } \\
\text { informativa dos custos correntes e a } \\
\text { adequação ao sistema existente. }\end{array}$ & $\begin{array}{l}\text { Administração } \\
\text { Federal } \\
\text { (Não específico) }\end{array}$ & 42 & 25 \\
\hline $\begin{array}{c}\text { Almeida, } \\
\text { Borba e } \\
\text { Flores } \\
(2009) \\
\end{array}$ & $\begin{array}{l}\text { Empírico } \\
\text { Descritivo }\end{array}$ & $\begin{array}{l}\text { São raros os casos de adoção e efetivo } \\
\text { uso da contabilidade de custos por parte } \\
\text { dos municípios da amostra analisada, } \\
\text { ainda que a LRF determine o contrário. }\end{array}$ & $\begin{array}{l}\text { Administração } \\
\text { Municipal } \\
\text { (Secretarias de } \\
\text { Saúde) }\end{array}$ & 12 & 0 \\
\hline $\begin{array}{l}\text { Gonçalves, } \\
\text { Zac e } \\
\text { Amorim } \\
(2009)\end{array}$ & $\begin{array}{l}\text { Empírico } \\
\text { Descritivo }\end{array}$ & $\begin{array}{l}\text { Descreve um estudo de apuração de } \\
\text { custos via Activity Based Costing. Os } \\
\text { resultados obtidos com o método } \\
\text { propiciarão à organização um controle } \\
\text { mais eficiente de custos, e capacitação } \\
\text { para certificações de gestão. }\end{array}$ & $\begin{array}{l}\text { Setor Hospitalar } \\
\text { (Estudo de caso } \\
\text { único) }\end{array}$ & 14 & 50 \\
\hline \multicolumn{6}{|c|}{$\begin{array}{l}\text { *Abordagem metodológica: "Prescritivo" denota um estudo que defende a adoção de um sistema, em } \\
\text { detrimento de outros, utilizando como argumentos pontos positivos não baseados em experiências prévias, ou } \\
\text { explicações teóricas sobre antecedentes ou implicações da adoção. O pesquisador está distante do objeto de } \\
\text { pesquisa, ou não utiliza a realidade particular das entidades para qual prescreve para analisar as consequiências } \\
\text { ou adoção. Utiliza sim um estereótipo das entidades, por vezes colocando mais ênfase em aspectos positivos } \\
\text { da adoção. "Empírico" denota a aproximação do pesquisador ao objeto de análise, tendo contato com a } \\
\text { realidade pesquisada, seja através de estudo de campo, da aplicação de questionários ou da realização de } \\
\text { entrevistas. "Descritivo" denota o compromisso do pesquisador em relatar a realidade observada sem } \\
\text { objetivar construir ou testar relações causais. } \\
\text { **Referências predominantes: " } K \text { " é a proporção de referências a artigos publicados em periódicos } \\
\text { científicos (peer reviewed) nacionais ou internacionais em relação ao total de referências. } \\
\text { **Referências predominantes (2): " } L " \text { é a proporção de referências a artigos publicados em periódicos } \\
\text { estrangeiros em relação ao total de referencias a artigos publicados em periódicos acadêmicos. }\end{array}$} \\
\hline
\end{tabular}

Quadro 1 - Estudos sobre aplicação de sistemas gerenciais de custos no setor público brasileiro publicados em periódicos nacionais 
Uma parte menor das fontes referenciadas é decorrente de periódicos, as quais apresentariam maior rigor no uso de teorias como base para estudos descritivos ou explicativos (ainda que o estudo de Lagioia et al. (2008) seja uma exceção). Mesmo estudos prescritivos, ao defenderem a adoção de um ou outro sistema, poderiam vir embasados em experiências anteriores de adoção de sistemas no setor público, ou mesmo no setor privado, como é o caso de Luque et al. (2008).

A ausência de suporte teórico inibe a discussão em torno das variáveis relevantes e relacionadas ao emprego de sistemas gerenciais de custos no setor público. Não ocorre a discussão das motivações dos agentes em relação à adoção e efetivo uso deste ferramental. Não se discute como, nem porque, diferentes níveis de elaboração de métricas de custeio são aplicados em determinados contextos. Tampouco é discutido como o papel estratégico de cada organização se relaciona com os sistemas aplicados, ou se estes refletem realmente a missão das entidades públicas analisadas. Questões de eficiência também são desconsideradas, visto não haver construtos teóricos definindo o que eficiência significa em cada contexto analisado. A própria questão da aderência das tecnologias de gestão derivadas da esfera privada (que vêm crescentemente sendo aplicadas às organizações públicas) permanece carente de análises profundas do ponto de vista teórico. Frisa-se que a efetividade dessa aderência tem sido questionada quanto à sua contribuição ao setor público (ITTNER; LARCKER, 1998; LAPSLEY, 2009).

\subsection{Análise crítica}

Machado (2005), com um dos livros mais relevantes na discussão da necessidade de mensuração de custos no setor público brasileiro, propõe um sistema de mensuração e uma estratégia de implantação do sistema de custos no setor público. No entanto, por ser um livro didático, não é aqui analisado em profundidade, uma vez que se optou por analisar artigos publicados em periódicos acadêmicos.

O primeiro estudo nacional voltado para a difusão de sistemas de custos na administração pública é de Alonso (1999). Neste trabalho o autor suscita a questão no meio acadêmico antes mesmo da publicação da LRF, e aborda as questões: (i) da mudança de paradigma do Estado gerencial (voltado para resultados) e (ii) a necessidade de desenvolvimento de sistemas de informação que indiquem eficiência no consumo de recursos (ao invés de seu simples registro). Nas palavras do autor, " o governo desconhece, em resumo, onde há desperdício e onde há eficiência na aplicação dos recursos. De fato, o modelo de gestão tradicional do setor público prioriza o controle dos recursos e dos procedimentos formais, e não os resultados, o desempenho, a eficiência". Apesar dos avanços iniciados no final da década de 1990 como a proposta pela LRF e a implantação do Sistema Integrado de Administração Financeira (SIAFI), o governo federal se ressentiria, da ausência de um sistema de apuração de custos configurado para subsidiar um desejado perfil gerencial e voltado para resultados.

No trabalho é proposto o emprego do Activity Based Costing (ABC) como meio para apuração e controle de custos, justificando a proposta, tanto pela grande quantidade de custos fixos indiretos como pelo perfil de prestador de serviços que caracteriza as diversas unidades organizacionais do governo. A grande conveniência da implantação do ABC - à época - seria o aproveitamento das informações de despesa fornecidas pelo SIAFI. A designação de tais despesas a atividades e objetos de custeio decorreria da modelagem característica de um projeto de $\mathrm{ABC}$.

No entanto, ao assumir o $\mathrm{ABC}$ como a melhor das possibilidades em termos de sistemas gerenciais de custos, o trabalho abre mão de discutir as necessidades do usuário da informação de custos. Independente do mérito da adequação ou eficiência do $\mathrm{ABC}$ como ferramenta de gestão, a sugestão de se aplicar uma ferramenta gerencial indiscriminadamente 
sugere um excesso de generalização. Trabalhos anteriores indicam que, mesmo na iniciativa privada, a taxa de adesão ao ABC tem diminuído em vários cenários (MALMI, 1997; INNES; MITCHELL, 1995). Estas pesquisas indicam que especificidades das firmas tornaram o benefício gerado pelo $\mathrm{ABC}$ aquém dos custos de sua adoção e/ou manutenção. Talvez tal generalização seja consequiência de debates ideológicos que ocuparam o cenário na academia nacional entre as escolas do custeio variável e do ABC na segunda metade da década de 1990.

Ao propor um determinado modelo como ideal ou ótimo, uma etapa fundamental do processo de escolha pode ser (e tem sido) negligenciado: a identificação de variáveis que antecedem a opção por um determinado modelo ou sistema gerencial baseado em custos. Diferentes perspectivas teóricas apontam para variáveis antecedentes relacionadas à opção, adoção e nível de sucesso de sistemas de controle gerencial (incluindo o controle de custos).

A indicação de um determinado modelo sem considerar as idiossincrasias de cada unidade do governo não tem recebido muita acolhida na comunidade científica. A razão deste questionamento se justifica pelo fato das unidades do governo ocuparem inúmeros setores da sociedade, cada qual com pacotes distintos de insumos, processos e produtos gerados para a sociedade. Variam ainda em termos de prioridade para verbas e projetos, seja por questões técnicas prioritárias ou da própria estrutura de poder (ocasional ou não). A literatura internacional já identificou e testou alguns destes fatores, subsidiando correntes teóricas em torno do tema (GEIGER; ITTNER, 1996). Como indicado pelos estudos nacionais levantados para este estudo, ainda não se caminhou neste sentido no Brasil.

O segundo estudo identificado é de Miranda et al. (2007), os quais levantaram informações acerca da aplicação e utilização do custeio $\mathrm{ABC}$ em hospitais universitários e compararam seus resultados com dados extraídos de outros estudos acerca do uso do ABC em outros setores econômicos. Foram enviados questionários para a direção de hospitais universitários (alguns privados) em busca dos dados que suportariam a análise. Segundo os autores foi verificada uma baixa adesão ao modelo $\mathrm{ABC}$ em função de seu alto custo e complexidade, além de não justificar o investimento (a informação presente já atenderia as expectativas). Chama mais a atenção o fato de que quase $70 \%$ dos respondentes desprezam a informação de custos.

Diferente de outros trabalhos sobre o $\mathrm{ABC}$ levantados para o presente artigo, este trabalho investiga o grau de adesão a esta ferramenta em diferentes contextos. Tal questionamento contribuiu positivamente para a discussão da adequação dos sistemas gerenciais, indicando a necessidade de serem consideradas variáveis de influência sobre a adesão e o sucesso de qualquer modelo.

No entanto, do ponto de vista da construção de teorias subjacentes, algumas questões ligadas ao uso da informação de custos não foram abordadas. Por exemplo, na amostra estavam presentes alguns hospitais particulares, o que potencialmente geraria uma fonte de comparação dentro da própria amostra quanto às diferenças em capacitação profissional da gestão, influência do corpo médico na tomada de decisões, entre outras.

Lagioia et al. (2008) avaliam os efeitos da padronização de processos e atividades sobre custos e qualidade de serviços em uma organização hospitalar. Após identificar um determinado procedimento cirúrgico corretivo como objeto de custeio, os autores coletaram dados históricos de custos anteriores e posteriores à implantação do projeto de padronização. Os resultados, segundo os autores, apontam para ganhos de desempenho em custos e qualidade do serviço prestado. Este é o trabalho com o maior uso de referências a artigos publicados em periódicos acadêmicos, especialmente a periódicos estrangeiros (vide colunas $\mathrm{K}$ e L do Quadro 1). Percebe-se no estudo que os autores buscaram contextualizar as atividades cobertas na análise, inclusive buscando literatura médica para isso, assumindo um caráter multidisciplinar ao abordar a otimização de processos com foco no ambiente de produção. 
Embora não usem derivações teóricas para suportar suas hipóteses ( $a d$ hoc), os autores aplicam abordagem quantitativa de teste de hipóteses para tentar validar estatisticamente as relações propostas entre padronização e desempenho. No entanto, a ausência de teoria impede o aprofundamento de questões relevantes. Por exemplo, segundo os autores, a gestão hospitalar vem recentemente desenvolvendo ferramentas para controlar a ação dos médicos no tocante ao consumo de recursos da organização. Estudos anteriores, que investigam a aplicação de sistemas gerenciais em hospitais, relacionaram a intensidade destes controles às características da gerência das unidades (ABERNETHY; VAGNONI, 2004; PIZZINI, 2006). Abernethy e Vagnoni (2004) identificaram controles mais rígidos sendo aplicados pela alta gerência em situações onde o corpo médico local exercia maior influência sobre a gestão da unidade. Por sua vez, ainda no contexto do setor hospitalar, Pizzini (2006) concluiu que a maior complexidade de processos nos hospitais leva à uma maior sofisticação da informação de custos, pela dificuldade em medir desempenho e distribuir responsabilidades.

Seguindo o levantamento da literatura nacional em torno do emprego de sistemas de custeio em órgãos públicos, Silva, Davis e Viveiros (2008) também mencionam uma reorientação de setores ligados ao governo em direção a um perfil mais gerencial, voltado para aspectos de eficiência e resultados. O estudo descreve um processo de reorganização da informação de custos na Marinha do Brasil. Segundo os autores, restrições orçamentárias incentivaram o comando administrativo da Marinha a buscar informações mais detalhadas do consumo de recursos de unidades de suporte e prestação de serviço interno (chamadas OMPS)

Em síntese, a Marinha buscou quantificar o montante de recursos orçamentários necessários para que estas unidades conseguissem manter-se em operação mesmo em períodos sem encomendas de produtos ou serviços por parte dos departamentos clientes. Tal montante recebeu o nome de Gestão de Posses Estratégicas (GPE). O texto afirma que a estrutura de informações pré-existente (no SIAFI e nas próprias OMPS) não atenderia essa necessidade específica do comando administrativo, de forma que foi necessário desenvolver um modelo de informação de custos próprio.

O estudo abre espaço para uma diferente perspectiva quanto ao conceito de eficiência e a necessidade de se mensurá-la. Aqui, a departamentalização e alocação de recursos são desenhadas para garantir a disponibilidade estratégica de unidades que lidam com demandas esporádicas, mas fundamentais para o exercício das atividades da Marinha do Brasil.

Os autores aplicam um estudo de caso único baseado em entrevistas com atores da organização estudada, e na coleta e interpretação de documentação. $\mathrm{O}$ estudo descreve o processo de implantação de conceitos advindos da contabilidade de custos em uma organização que não dispunha de informações detalhadas de seus custos. Os autores classificam o estudo como exploratório, e desconsideram estudos estrangeiros antecedentes, tendo referências e citações concentradas em livros textos.

Chama a atenção neste e em outros trabalhos analisados nesta pesquisa a recorrente retórica da falta de literatura em torno do uso de sistemas gerenciais de custos no setor público. Se por um lado, de fato, a literatura nacional desprezou tal fenômeno, o mesmo não pode ser dito para a literatura internacional. Por exemplo (e contextualizando a análise do trabalho), Ansari e Euske (1987) analisam a utilização da informação de custo nas instalações do exército dos EUA e verificam influências institucionais sobre sistemas de custeio. Ou seja, as informações de custos são úteis para legitimar a qualidade de gestão, mas pouco empregadas para o processo decisório local.

Almeida, Borba e Flores (2009) analisam o uso da informação de custos pelas secretarias municipais de saúde em municípios do Estado de Santa Catarina. Através de questionários enviados aos órgãos de saúde, os autores procuraram identificar se existe algum controle de custos nestas secretarias. Segundo os resultados do estudo de caso, das 20 secretarias analisadas apenas duas apresentaram algum nível de formalização na gestão de 
custos. Este quadro segue em direção oposta às determinações da LRF. No caso em questão, quase $70 \%$ dos entrevistados exerciam a posição de gestores há menos de um ano - no momento da condução da pesquisa. Contudo, o estudo não aponta se existe alguma relação entre o nível de profissionalismo da gestão destas secretarias, o tempo destes gestores no cargo, e o emprego de ferramentas formais de controle.

O desenvolvimento de teorias a partir de estudos de caso apresenta vantagens potenciais quanto à tempestividade, testabilidade de hipóteses e a validação da pesquisa que surgem a partir da ligação íntima entre o estudo e a evidência empírica (EISENHARDT, 1989). No entanto, poucos avanços são esperados caso os estudos se restrinjam continuamente a apenas descrever o estado da arte sem buscar o entendimento das variáveis antecedentes que o geraram; ou ainda, buscar traçar possíveis conseqüências do estágio atual. No caso analisado (ALMEIDA; BORBA; FLORES, 2009) os autores levantam um interessante volume de dados dos organismos pesquisados, mas abdicam da oportunidade de verificar se modelos teóricos previamente desenvolvidos são aderentes à realidade nacional.

Estudos anteriores aplicando surveys em órgãos de governo identificaram se fatores organizacionais têm uma influência positiva significativa sobre o desenvolvimento do sistema de mensuração, entre estes fatores estão o comprometimento da alta gerência com a utilização de informações de desempenho, o poder de decisão e a formação de técnicas de medição de desempenho (CAVALUZZO; ITTNER, 2004). Este é um exemplo típico do poder de informação contido em dados de governo quanto ao emprego de artefatos gerenciais que poderia orientar esforços de pesquisa futura.

Luque et al. (2008) seguem uma linha similar à de Alonso (1999) com relação à prescrição de um modelo de custos aplicável ao governo federal. No entanto, ao invés de propor o ABC, cujas perspectivas de aquisição e manutenção são de elevado custo, é proposto um modelo híbrido de custeio baseado nas principais contas de custo corrente do governo (pessoal da ativa e insumos intermediários). O termo híbrido (adotado aqui) refere-se ao fato de serem considerados os custos de capital fixo quando os mesmos forem relevantes e vinculáveis aos objetos de custeio. Os principais benefícios seriam dispor de capacidade informativa dos custos correntes e adequação ao sistema de informação existente. Desta forma (segundo os autores), o modelo manteria as propriedades alocativas desejadas, além de ser adaptado ao sistema de informações presente no sistema SIAFI. O principal objetivo do trabalho "é o de explicitar uma sistemática de análise e apuração de custos de serviços públicos que seja adaptada ao próprio processo orçamentário do setor público brasileiro e, assim, contribuir para a obtenção da eficiência alocativa e operacional".

O estudo também cita a LRF e a tendência de um perfil mais gerencial e menos burocrático do Estado como justificativas para que o governo federal invista em sua contabilidade de custos. Pelo observado nas experiências internacionais, é bem vinda a proposta de formalizar mecanismos gerenciais de forma que os mesmos sejam aderentes, tanto à estrutura física presente (sistemas de informações) quanto às diversas complexidades da dinâmica administrativa do setor público.

Também neste estudo percebe-se o mesmo viés prescritivo e generalista de outros trabalhos em torno do tema. Uma solução operacional para gestão de custos é proposta sem que se discuta o nível de utilidade percebida pelos agentes da informação de custos em questão. Entidades tão díspares como bancos, companhias de produção e distribuição de petróleo e derivados, universidades, agências reguladoras e tantas outras usam a informação de custos de forma bastante diversa, demandando sistemas diferentes, variações dentro do mesmo sistema e assim por diante.

Faz referência a uma quantidade razoável de artigos publicados em periódicos acadêmicos, um quarto deles publicado em periódicos estrangeiros. Neste trabalho são discutidos insights históricos relacionados à avaliação de desempenho do gestor público, 
como, por exemplo, a Lei do PPA 2000-2003 e o Decreto n 2.829, de 1998, além da própria LRF, de 2000. Tais estímulos poderiam indicar direções para pesquisas futuras sobre a influência institucional na adoção de sistemas gerenciais distintos.

No entanto, do ponto de vista de cada entidade do governo, caberia entender como os diferentes níveis de coerção desta legislação afetariam o emprego e efetiva utilização da informação de custo. Por exemplo, estudos anteriores indicaram que unidades do governo dos EUA mais expostas à competição por fundos federais investiram em sistemas mais sofisticados de custeio, na intenção de sinalizar qualidade em gestão e com isso atrair uma fatia maior do orçamento (GEIGER; ITTNER, 1996; ITTNER; LACKER, 1998).

O último trabalho analisado é Gonçalves, Zac e Amorim (2009). Os autores procuram captar as potencialidades (positivas) da adoção do custeio $\mathrm{ABC}$ em um hospital da rede pública no Estado de Minas Gerais. A opção pelo ABC seria desejável para reorientar estrategicamente a tomada de decisão da organização. No caso, a organização se beneficiaria com o redesenho de seus processos e atividades de forma a atingir dois objetivos principais: (i) alcançar liderança em custos no setor e (ii) tornar-se apta à certificação "Acreditação Hospitalar (ONA)". O estudo de caso lança mão de entrevistas e análise de documentação para verificar a condição presente de apuração de custos na organização. Após selecionar um setor do hospital e um procedimento cirúrgico como objeto de custeio, os autores simulam a aplicação do ABC. Com o detalhamento dos processos e identificação dos recursos consumidos, mapeamentos (atividades e direcionadores) e estrutura de custo são obtidos.

Os autores indicam que o artigo abordou uma fase de um projeto de redesenho organizacional de uma organização. O estudo sugere, sem maiores evidências, que o método ABC seria o mais adequado, por detalhar mais a informação de custos e proporcionar um recapeamento de processos e atividades. As referências do estudo não contemplam a literatura internacional, e se concentram em livros textos em administração e contabilidade. Nenhuma proposição teórica é considerada.

No entanto, o artigo contribui no sentido em que uma ferramenta gerencial é aplicada a relacionar duas propostas teóricas em torno do tema: a teoria da contingência e a teoria institucional. Quando os autores mencionam que o $\mathrm{ABC}$ é interpretado como adequado para um re-mapeamento eficiente de processos, uma oportunidade é aberta para se investigar a proposta da teoria da contingência, a qual propõe que sistemas gerenciais evoluem de acordo com a identificação de oportunidades de melhoria pelos atores organizacionais (CHENHALL, 2003). Por outro lado, é mencionado que o ABC seria útil ao hospital analisado para melhorar seus procedimentos de forma a obter uma certificação. Essa colocação corrobora a proposta da teoria institucional de que sistemas gerenciais podem evoluir para atender expectativas de atores externos à organização e, assim, servir a sua legitimação (ITTNER; LACKER, 1998).

\section{CONCLUSÕES}

Buscando sumarizar a literatura nacional de sistemas de custos aplicada ao setor público, este trabalho procurou regularidades nas contribuições propostas em cada estudo, assim como as abordagens teóricas e as estratégias de pesquisa adotadas nestes estudos. $\mathrm{Na}$ busca realizada em periódicos acadêmicos nacionais, identificou-se a (escassa) literatura nacional sobre o tema, consolidando alguns resultados apresentados sobre organizações similares (hospitais públicos).

Pode-se sumarizar os estudos analisados em iniciativas que defendem a implantação deste ou daquele modelo, e iniciativas que adotam uma abordagem descritiva de sistemas isolados. Contrariando o alerta da ameaça à construção de conhecimento da temática provocada pela falta de sistematização dos resultados e a desconsideração de estudos anteriores no tema (ZIMMERMAN, 2001; LUKKA; MOURITSEN, 2002; HOPWOOD, 2002; LUFT; SHIELDS, 2002), a literatura nacional está carente de comparação tanto com 
estudos estrangeiros, quando com iniciativas nacionais. Não se encontrou nenhum estudo de caso comparado. Embora alguns estudos no exterior sinalizem um caminho para investigação científica, através do uso de levantamentos comparando os efeitos de variáveis de contexto e institucionais, no Brasil tal caminho permanece pouco percorrido. As poucas pesquisas encontradas restringem-se a descrever o quadro ou a prescrever soluções, abdicando-se de desafiar teorias. O estágio inicial de descrição, sem comparação, e sem embasamento teórico mostra que se está no início da pesquisa do tema no Brasil. Contudo, apesar das limitações listadas, há que se reconhecer a importância destas iniciativas de pesquisa para desenvolvimento de teoria no contexto nacional.

O Brasil está passando por um momento único em termos de adoção de sistemas de custos no setor público. Os estímulos da LRF possivelmente motivaram e motivarão a contratação de desenvolvimento de projetos de implantação de métricas de orçamento e custeio em inúmeros órgãos, autarquias, empresas estatais e fundações em todo o país. $\mathrm{O}$ Ministério da Fazenda tem conduzido seminários estimulando o debate, a reflexão e a sensibilização de agentes-chave no processo de implantação de sistemas de informação de custos no setor público, assim como o desenvolvimento de procedimentos de coleta de dados relativos à liquidação de despesas, seus ajustes ao regime de competência e os procedimentos para transformá-los em informações de custos.

Ações como esta, potencialmente lançam oportunidade para: (i) comparar a variação dos critérios de escolha dos diversos artefatos gerenciais demandados; (ii) avaliar o impacto da motivação orçamentária ou das demandas dos órgãos de controle; e (iii) estimar o real grau de relevância e utilidade percebida pelos gestores envolvidos na operação e manutenção dos sistemas de informação.

Diversas questões continuam em aberto, representando uma ampla agenda de pesquisa a ser desenvolvida em âmbito nacional e que parece ser de interesse de periódicos nacionais e internacionais. Por exemplo, quais os fatores que conduzem à adoção isolada por determinadas entidades? Quais fatores direcionam a escolha de determinadas métricas (sistemas, metodologias etc.) pelas unidades que se lançaram na iniciativa de mensuração de custos? Quais obstáculos justificariam determinadas entidades a não colocarem esforços na mensuração de custos?

Como dito, o setor público apresenta diferenças em relação ao setor privado, que por si só demanda grande aprofundamento de análise e adequação de hipóteses que explicariam a adoção e uso de sistemas de custos. Entre elas pode-se citar: (i) a dificuldade em identificar objetivos apropriados em ambientes caracterizados por metas individuais conflitantes, (ii) mensurar o desempenho em tarefas altamente subjetivas, (iii) prover incentivos eficientes aos diversos agentes, considerando as instituições de isonomia e estabilidade, (iv) restrições de tecnologia disponível nas diversas entidades públicas, assim como a capacitação para contratá-la ou gerenciá-la afeta o sucesso das inovações propostas para o sistema de informação corrente (CAVALLUZZO; ITTNER 2004), (v) as diferenças de definição ou de formato da estrutura da informação, por vezes oriundas de diferentes bases espalhadas por órgãos estatais, e potenciais tensões de padronização e migração de dados (KRAVCHUK; SCHACK 1996).

\section{REFERÊNCIAS}

ABERNETHY, M.; VAGNONI, E. Power, organization design and managerial behaviour. Accounting, Organizations and Society, n. 29, n. 3-4, p. 207-225, Apr./May, 2004. http://dx.doi.org/10.1016/S0361-3682(03)00049-7

ALMEIDA, A.; BORBA, J.; FLORES, L. A utilização das informações de custos na gestão da saúde pública: um estudo preliminar em secretarias municipais de saúde do estado de Santa 
Catarina. Revista da Administração Pública, v. 43, n. 3, p. 579-607, maio/jun., 2009.

ALONSO, M. Custos nos serviços públicos. Revista do Serviço Público, n. 50, p. 37-54, 1999.

ANSARI, S.; EUSKE, K. Rational, rationalizing, and reifying uses of accounting data in organizations. Accounting, Organizations and Society, v. 12, n. 6, p. 549-570, 1987. http://dx.doi.org/10.1016/0361-3682(87)90008-0

AQUINO, A.; PAGLIARUSSI, M.; BITTI, E. Heurística para composição de referencial teórico. Revista Contabilidade e Finanças, v. 19, n. 47, p. 73-88, maio/ago., 2008. http://dx.doi.org/10.1590/S1519-70772008000200007.

BAMBER, L.; CRISTENSEN, T.; GAVER, K. Do we really 'know' what we think we know? A case study of seminal research and its subsequent overgeneralization. Accounting, Organizations and Society, v. 25, n. 2, p.103-129, Feb., 2000. http://dx.doi.org/10.1016/S0361-3682(99)00027-6

BHIMANI, A. (org.) Management Accounting: European perspectives. Oxford: Oxford University Press, 1996.

BJØRNENAK, T. Understanding cost differences in the public sector: a cost drivers approach. Management Accounting Research, v. 11, n.2, p. 193-211, 2000. http://dx.doi.org/10.1006/mare.2000.0128

BROWN, L. The importance of circulating and presenting manuscripts: evidence from the accounting literature. The Accounting Review. v. 80, n. 1, p. 55-83, 2005. http://dx.doi.org/10.2308/accr.2005.80.1.55

BROWN, L.; GARDNER, J.; VASARHELY, M. An analysis of the research contributions of Accounting Organizations and Society, 1976-1984. Accounting, Organizations and Society. v. 12, n. 2, p. 388-397, 1987. http://dx.doi.org/10.1016/0361-3682(87)90006-7

BROWN, R.; SPROGHE, H. Governmental managerial accounting: What and where is it? Public Budgeting and Finance, v. 7, n. 3, p. 35-46, Oct., 1987. http://dx.doi.org/10.1111/1540-5850.d01-230

CARDOSO, R.; OYADOMARI, J.; MENDONÇA NETO, O. A influência da positive accounting nos programas de mestrado em contabilidade: uma análise bibliométrica da produção acadêmica de 2002 a 2005. Brazilian Business Review, v. 4, n. 2, p. 158-170, maio/ago., 2007.

CARDOSO, R.; MENDONÇA NETO, O.; RICCIO, E.; SAKATA, M.. Pesquisa científica em contabilidade entre 1990 e 2003, RAE. Revista de Administração de Empresas, v. 45, n. 2, abr./jun., 2005. http://dx.doi.org/10.1590/S0034-75902005000200004.

CAVALLUZZO, K.; ITTNER, C. Implementing performance measurement innovations: evidence from government. Accounting Organizations and Society, v. 29, p. 243-267, 2004. http://dx.doi.org/10.1016/S0361-3682(03)00013-8

CHENHALL, R. Management control systems design within its organizational context: findings from contingency-based research and directions for the future. Accounting, Organizations and Society, v. 28, n. 2-3, p. 127-168, 2003. http://dx.doi.org/10.1016/S03613682(01)00027-7

DIMAGGIO, P.; POWELL, W. The iron cage revisited: institutional isomorphism and collective rationality in organizational fields. American Sociological Review, v. 48, n.2, p. 147-160, Apr., 1983. http://dx.doi.org/10.2307/2095101 
DRAZIN, R.; VAN de VEN, A. Alternative forms of fit in contingency theory. Administrative Science Quarterly, v. 30, n. 4, p. 514-539, Dec., 1985. http://dx.doi.org/10.2307/2392695

EISENHARDT, K. Building theories from case study research. The Academy of Management Review, v. 14, n.4, p. 532-550, Oct., 1989. http://dx.doi.org/10.2307/258557

FREZATTI, F. Análise dos traços de tendências de uma amostra das revistas científicas da área de contabilidade publicadas na língua inglesa. Caderno de Estudos/FIPECAFI, v. 13, n. 24, p. 50-78, jul./dez., 2000.

GEIGER, D.; ITTNER, C. The influence of funding source and legislative requirements on government cost accounting practices. Accounting Organizations and Society, v. 21, n. 6, p. 549-567, Aug., 1996. http://dx.doi.org/10.1016/0361-3682(96)00008-6

GONÇALVES, M.; ZAC, J.; AMORIM, C. Gestão estratégica hospitalar: aplicação de custos na saúde. Revista de Administração FACES Journal, v. 8, n. 4, p. 161-179, out./dez., 2009.

GUPTA, P.; DIRSMITH, M.; FOGARTY, T. Coordination and control in a government agency: contingency and institutional theory perspectives on GAO audits. Administrative Science Quarterly, v. 39, n. 2, p. 264-284, Jun., 1994. http://dx.doi.org/10.2307/2393236

HARTMANN, F. The appropriateness of RAPM: toward the further development of theory. Accounting, Organizations and Society, v. 25, n. 4-5, p.451-482, Apr./May, 2000. http://dx.doi.org/10.1016/S0361-3682(98)00036-1

HILL, N. Adoption of costing systems in U.S. hospitals: an event history analysis 1980-1990. Journal of Accounting and Public Policy, v. 19, n. 1, p. 41-71, 2001. http://dx.doi.org/10.1016/S0278-4254(99)00013-7

HOPWOOD, A. If only there were simple solutions, but there aren't: some reflections on Zimmerman's critique of empirical management accounting research. The European $\begin{array}{lllllll}\text { Accounting Research, } & \text { v. 11, n. 4, p. } & 777-785, & \end{array}$ http://dx.doi.org/10.1080/0963818022000047073

HULL, R.; WRIGHT, G. Faculty perceptions of journal quality: an update. Accounting Horizons, v. 4, n. 1, p. 77-98, Mar., 1990.

INNES, J.; MITCHELL, F. A survey of activity-based costing in the U. K.'s largest companies. Management Accounting Research, v. 6, n. 2, p. 137-153, 1995. http://dx.doi.org/10.1006/mare.1995.1008

ITTNER, C.; LARCKER, D. Innovations in performance measurement: trends and research implications. Journal of Management Accounting Research, v. 10, p. 205-238, 1998.

ITTNER, C.; LARCKER, D. Assessing empirical research in managerial accounting: a valuebased management perspective. Journal of Accounting and Economics. v. 32, p. 349- 410, 2001. http://dx.doi.org/10.1016/S0165-4101(01)00026-X

ITTNER, C.; LARCKER, D. Empirical managerial accounting research: are we just describing management consulting practice? The European Accounting Research, v. 11, n. 4, p.787-794, 2002. http://dx.doi.org/10.1080/0963818022000047082

KRAVCHUK, R.; SCHACK, R. Designing effective performance-measurement systems under the government performance and results act of 1993. Public Administration Review, v. 56, p. 348-358, 1996. http://dx.doi.org/10.2307/976376

LAGIOIA, U.; RIBEIRO, J.; FALK, J.; LIBONATI, J.; LOPES, J. A gestão por processos gera melhoria de qualidade e redução de custos: o caso da unidade de ortopedia e 
traumatologia do Hospital das Clínicas da Universidade Federal de Pernambuco. Revista de $\begin{array}{lllllllll}\text { Contabilidade } & \text { e Finanças, v. 19, n. 48, p. 77-90, } & \text { set./dez., } 2008 .\end{array}$ http://dx.doi.org/10.1590/S1519-70772008000300007.

LANGFIELD-SMITH, K. Management control systems and strategy: a critical review. Accounting, Organizations and Society, v. 22, n. 2, p. 207-232, Feb., 1997. http://dx.doi.org/10.1016/S0361-3682(95)00040-2

LAPSLEY I.; WRIGHT, E. The diffusion of management accounting innovations in the public sector: a research agenda. Management Accounting Research, v. 15, n. 3, p. 355374, Sep., 2004. http://dx.doi.org/10.1016/j.mar.2003.12.007

LAPSLEY, I. New public management: the cruellest invention of the human spirit? Abacus, v. 45, n. 1, p. 1-21, 2009. http://dx.doi.org/10.1111/j.1467-6281.2009.00275.x

LAWRENCE, C. The effect of ownership structure and accounting system type on hospital costs. Research in Governmental and Nonprofit Accounting, v. 6, p. 35-60, 1990.

LUFT, J.; SHIELDS, M. Zimmerman's contentious conjectures: describing the present and prescribing the future of empirical management accounting research. The European $\begin{array}{llllllll}\text { Accounting Review, } & \text { v. } 11, \quad \text { n. } 4, & \text { p. } & 795-805, & \end{array}$ http://dx.doi.org/10.1080/0963818022000047091

LUFT, J.; SHIELDS, M. Mapping management accounting: graphics and guidelines for theory-consistent empirical research. Accounting, Organizations and Society, v. 28, n. 2-3, p. 169-249, Feb./Apr., 2003. http://dx.doi.org/10.1016/S0361-3682(02)00026-0

LUKKA, K.; GRANLUND, M. The fragmented communication structure within the accounting academy: the case of activity-based costing research genres. Accounting, Organizations and Society, v. 27, n. 1-2, p. 165-190, Jan./Mar., 2002. http://dx.doi.org/10.1016/S0361-3682(00)00037-4

LUKKA, K.; MOURITSEN, J. Homogeneity or heterogeneity of research in management accounting? The European Accounting Review, v. 11, n. 4, p. 805-811, 2002. http://dx.doi.org/10.1080/0963818022000047109

LUQUE, C.; CRUZ, H.; AMARAL, C.; BENDER, S.; SANTOS, P. O processo orçamentário e a apuração de custos de produtos e serviços no setor público do Brasil. Revista do Serviço Público, v. 59, n. 3, p. 309-331, jul./set., 2008.

MACHADO, N. Sistema de informação de custo: diretrizes para integração ao orçamento público e à contabilidade governamental. Brasília: ENAP, 2005.

MALMI, T. Towards explaining activity-based costing failure: accounting and control in a decentralized organization. Management Accounting Research, v. 8, p. 459-480, Apr., 1997. http://dx.doi.org/10.1006/mare.1997.0057

MARTINS, E. Contabilidade de custos. São Paulo: Atlas. 2006.

MERCHANT, K.; SIMON, R. Research and control in complex organizations: an overview. Journal of Accounting Literature, v. 5, p.183-203, 1986.

MIRANDA, G.; CARVALHO, C.; MARTINS, V.; FARIA, A. Custeio ABC no ambiente hospitalar: um estudo nos hospitais universitários e de ensino brasileiros. Revista de Contabilidade e Finanças, v. 44, n. 18, p. 33-43, maio/ago., 2007. http://dx.doi.org/10.1590/S1519-70772007000200004.

MURCIA, F.; BORBA, J. Possibilidades de inserção da pesquisa contábil brasileira no cenário internacional: uma proposta de avaliação dos periódicos científicos de contabilidade e 
auditoria publicados em língua inglesa e disponibilizados no portal de periódicos da CAPES. Revista de Contabilidade \& Finanças, v. 19, n. 46, p. 30-43, jan./abr., 2008. http://dx.doi.org/10.1590/S1519-70772008000100004

OLIVEIRA, M. Análise dos periódicos brasileiros de contabilidade. Revista Contabilidade \& Finanças, n. 29, p. 68-86, maio/ago., 2002. http://dx.doi.org/10.1590/S151970772002000200005 .

PACKER, A.; BIOJONE, M.; IRATI, A.; TAKENAKA, R.; GARCÍA, A.; SILVA, A.; MURASAKI, R.; MYLEK, C.; REIS, O.; DELBUCIO, H. SciELO: uma metodologia para publicação eletrônica. Ciência da Informação, v. 27, n. 2, p. 109-121, 1998.

PIZZINI, M. The relation between cost-system design, managers' evaluations of the relevance and usefulness of cost data, and financial performance: an empirical study of US hospitals. Accounting, Organizations and Society, v. 31, n. 2, p.179-210, 2006. http://dx.doi.org/10.1016/j.aos.2004.11.001

REITER, S.; WILLIANS, P. The structure and progressivity of accounting research: the crisis in the academy revisited. Accounting, Organizations and Society, v. 27, n. 2, p. 575-607, Aug., 2002 . http://dx.doi.org/10.1016/S0361-3682(01)00050-2

RICCIO, E.; CARASTAN, J.; SAKATA, M. Accounting research in Brazilian universities: 1962-1999. Caderno de Estudos/FIPECAFI, v. 11, n. 22, p. 35-44, set./dez., 1999.

SHIELDS, M. Management accounting practices in Europe: A perspective from the States. Management Accounting Research, v. 9, n. 4, p. 205-513, Dec., 1998. http://dx.doi.org/10.1006/mare.1998.0081

SILVA, A.; OLIVEIRA, E.; RIBEIRO FILHO, J. Revista Contabilidade \& Finanças - USP: uma comparação entre os períodos 1989/2001 e 2001/2004. Revista Contabilidade \& Finanças, v. 39, p. 20-32, set./dez., 2005. http://dx.doi.org/10.1590/S151970772005000300003.

SILVA, A.; DAVIS, M.; VIVEIROS, A. Contabilidade de custos na administração pública: o estabelecimento dos gastos de posse estratégica na marinha do Brasil. Revista do Serviço Público, v. 59, n. 4, p. 421-440, out./dez., 2008.

SILVA, C. (org.). Custos no setor público. Brasília: Editora Universidade de Brasília, 2007.

SPEKLÉ, R. Explaining management control structure variety: A transaction cost economics perspective. Accounting, Organizations and Society, v. 26, n. 4-5, p. 419-441, May/Jul., 2001. http://dx.doi.org/10.1016/S0361-3682(00)00041-6

THEÓPHILO, C.; IUDÍCIBUS, S. Uma análise crítico-epistemológica da produção científica em contabilidade no Brasil. UnB Contábil, v. 8, n. 2, jul./dez., 2005.

YAKHOU, M.; DORWEILER, V. Advanced cost management systems: an empirical comparison of England, France, and the United States. Advances in International Accounting, v. 8, p. 99-127, 1995.

ZIMMERMAN, J. Accounting for decision making and control. Boston: Irwin/McGraw Hill, 1997.

ZIMMERMAN, J. Conjectures regarding empirical managerial accounting research. Journal of Accounting and Economics, v. 32, p. 411-427, 2001. http://dx.doi.org/10.1016/S01654101(01)00023-4 